\title{
Refranes meteorológicos del asturianoleonés en su contexto romance, según los datos de ParemioRom ${ }^{1 /}$ Asturian meteorological sayings in their romance context, according to ParemioRom data
}

\author{
JOSÉ ENRIQUE GARGALLO GIL \\ UNIVERSITAT DE BARCELONA \\ INSTITUT D'ESTUDIS CATALANS
}

\begin{abstract}
Resume: El proyeutu ParemioRom (Paremiología romance: refranes meteorológicos $y$ territorio), anque peracabáu oficialmente hai unos años (FFI2011-24032), sigue calteniendo una vixencia científica dafechu. La so base de datos, qu'inxer sobro otra anterior (Base de datos sobre refranes del calendario y meteorológicos en la Romania: $B A D A R E$, 2005-2011) la funcionalidá de la xeolocalización, amiesta esti tipu de búsqueda posible a otres cuatro preexistentes de BADARE: testual, por llingües, temática (cronoloxía, meteoroloxía y ámbitu temáticu xeneral), y per fontes bibliográfiques.
\end{abstract}

La base de datos de ParemioRom permítenos ufrir una amuesa de les 726 fiches de refranes meteorolóxicos (paremies con implicación meteorolóxica o atmosférica) axuntaos pela etiqueta d'asturleonés; refranes que se localicen na so mayor parte n'Asturies, pero que s'allarguen tamién a Lleón. Y d'igual mou, la confrontación romance coles paremies homólogues o asemeyaes. Too ello, a partir de l'astraición, metodolóxicamente operativa, del conceutu de tipu parémicu o paremiotipu.

L'amuesa xébrase per estayes temátiques: altura y proyeición del sol, meses, feches fixes, meteoros varios; y permite alvertir semeyances de diversu calter col contestu románicu: davezu col oeste peninsular (gallegu y portugués), dacuando col conxuntu iberorromance, del que, significatiblemente, el catalán suel quedar al marxe. Danse tamién casos d'integración asturianolleonesa nuna Romania parémica continua, qu'abarca los diversos dominios del continuum territorial européu (más les islles mediterranees de la so contorna); y, sicasí, xebra al rumanu, llingua separtadiza non solo n'aspeutos de la so fisonomía romance, sino tamién no parémico y no folclórico. Del Atlas de ParemioRom (APR) ufiértase finalmente'l paremiotipu que respuende a la fórmula «[Si / cuando] la Candelaria llora [llueve] $\rightarrow$ invierno fuera; [si / cuando] la Candelaria ríe [sol] $\rightarrow$ invierno por venir», cola representación asturiana nel so contestu iberorromance.

Pallabres clave: paremioloxía, llingües romances, ParemioRom, Asturies.

Abstract: Even though the Project ParemioRom (Romance Paremiology: sayings and proverbs on meteorological issues and territory) finished some years ago (FFI2011-

${ }^{1}$ Agradezco las valiosas observaciones de Maria-Reina Bastardas y Joan Fontana. 
24032), it is still valid from a scientific point of view. Its data basis, which is inserted in a previous one (Database of calendar and meteorological sayings in the Romancespeaking areas: BADARE, 2005-2011), the functionality of its geolocalization brings together other four pre-existing search types: textual, according to language, thematic (chronology, meteorology and general) and according to bibliographical sources.

The ParemioRom database allows us to have a simple of 726 files of meteorological sayings (paroemies with meteorological or atmospheric implications), organised according to the Astur-Leonese label, proverbs which are mostly located in Asturias, but which extend to Leon; and, likewise, the Romance confrontation between similar paroemies will be shown. Methodologically, all these proverbs will be presented from the abstract concept of what is paremiological.

The sample is internally categorised according to thematic varieties such as sun height and projection, fixed dates or various atmospheric phenomena. It also shows similarities of different kind in the Romance-speaking context, specially with Western Galician and Portuguese, at times with the whole Iberian-Romance territory, from which Catalan is usually left apart. Besides, there are also some Asturian-Leonese cases which integrate into a Romance-speaking paremiological continuum, which extends along the diverse European territorial continuum (including the Mediterranean isles), and, in spite of this, far from Romanian, a different language not only in terms of aspect, but also in paremiological and folkloric aspects.

The Atlas de ParemioRom (APR) also offers a paremiotype which replies to the formula: «[If/when] the Candlemas cries [rains] $\rightarrow$ Winter is out; [if/when] the Candlemas smiles [shines] $\rightarrow$ Winter still to come», with its corresponding Asturian representation in the Iberian-Romance context.

Keywords: paremiology, Romance languages, ParemioRom Asturias.

\section{PAREMIOROM}

En la estela de lo que fue proyecto y, años después, cabe contemplar más bien como post-yecto, pues ya no tiene vigencia oficial, la mirada actual a la base de datos de ParemioRom ${ }^{2}$ constata, en cambio, que su vigencia científica y su utilidad para quien se interese por el mundo de los refranes (particularmente los meteorológicos) siguen siendo plenas.

ParemioRom (2011-2015) siguió el surco abierto por dos proyectos (¿post-yectos?) desarrollados bajo el emblema o acrónimo de BADARE (Base de datos sobre refranes del calendario y meteorológicos en la Romania, $2005-$ 2008, 2008-2011). Y con respecto a estos incorporó una funcionalidad que constituye aún hoy la principal innovación de su base de datos: a saber, la

${ }^{2}$ Acrónimo de Paremiología romance: refranes meteorológicos y territorio (FFI2011-24032), proyecto financiado por el Ministerio de Economía y Competitividad entre los años 2011 y 2015. Su investigador principal, o IP, fue quien suscribe. 
geolocalización aplicada a las fichas de refranes que ofrecen una precisión local de uso, lo que permite búsquedas simples o combinadas de múltiples tipos. Dicha funcionalidad posibilitó la realización de un Atlas de ParemioRom (APR), que es el destilado final de una década prodigiosa (2005-2015) de colaboración investigadora. ${ }^{3}$

La base de datos de ParemioRom, consultable a través del enlace $<$ http://stel.ub.edu/paremio-rom/es/refranes $>$ (en español, catalán o inglés), contiene 13215 fichas de refranes; y digo «fichas de refranes», y no «refranes», porque muchas de esas fichas acogen variantes, generalmente leves, que muy bien podrían verse como refranes distintos, y como tales se contarían sin duda en no pocos repertorios paremiográficos al uso. En cambio, nuestra base los reúne bajo una sola ficha. Y por más que la delimitación entre variantes y tipos principales de refrán no siempre resulta sencilla, aquellas son posibles candidatas a engrosar lo que en nuestro equipo denominamos tipo parémico o paremiotipo, ${ }^{4}$ que en la Presentación del APR se define así: ${ }^{5}$

Sobre el concepto de paremiotipo, cabe señalar que lo entendemos a manera de fórmula o abstracción bajo la que se acogen refranes similares en su estructura formal y en su contenido semántico, aun a sabiendas del carácter flexible de tales factores en algunos casos.

Son cinco los tipos de búsquedas: (1) textuales; (2) por lenguas y variedades; 38 en total, dentro de las cuales se incluye la etiqueta de asturleonés; ${ }^{6}$ (3) conceptuales, que se distribuyen en los bloques de Cronología, Meteorología y Ámbito temático general, y a su vez acogen categorías (como la meteorológica de cielo) y subcategorías (como arreboles); (4) por fuentes bibliográficas, como el Refranero asturiano de Castañón (1962a) o el DGLA; (5) por geolocalizaciones. Las fichas de geolocalización son 2633, de las cuales 260 corresponden a Asturias, y 169 a León. ${ }^{7}$

\footnotetext{
${ }^{3}<$ http://stel.ub.edu/paremio-rom/es/atlas $>$. Firman su Presentación en febrero de 2016, junto al autor de este artículo, Maria-Reina Bastardas, Joan Fontana y Antonio Torres, de la Universidad de Barcelona. Los cuatro colaboradores de la UB constituíamos inicialmente el núcleo fundacional del equipo investigador, al que se sumó en 2012 Xosé Afonso Álvarez, entonces adscrito al Centro de Linguística da Universidade de Lisboa (CLUL), y ahora (2019) a la Universidad de Alcalá.

4 Quien realice una búsqueda entrecomillada de ambas secuencias, «tipo parémico» y «paremiotipo», en Google comprobará las múltiples citas derivadas de nuestras publicaciones.

$5<$ http://stel.ub.edu/paremio-rom/es/atlas/atlas-de-paremiorom-apr-presentaci\%C3\%B3n $>$.

${ }^{6}$ La etiqueta de asturleonés utilizada en ParemioRom responde al uso normativo que prescribe el $D L E$ para el castellano. En este artículo, en cambio, me acojo ya desde el título a la designación de asturianoleonés, más propia de Lletres Asturianes y de su entorno académico.

${ }^{7}$ Una mayoría de las 260 fíchas de geolocalización de Asturias se asigna a localidades de habla asturiana. Por lo que respecta a las 169 de León, la proporción de lugares que no son estrictamente de habla leonesa es sensiblemente mayor.
} 
Son 726 las fichas de refranes con la etiqueta de asturleonés, y se localizan mayoritariamente en Asturias. Por último, y por desgracia, nuestra empresa no alcanzó para incluir datos del mirandés.

A manera ilustrativa, se ofrece en la figura 1 el resultado de la búsqueda de lenguas y variedades $>$ asturleonés, que anuncia 726 fichas de refranes.

\section{Fichas de refranes}

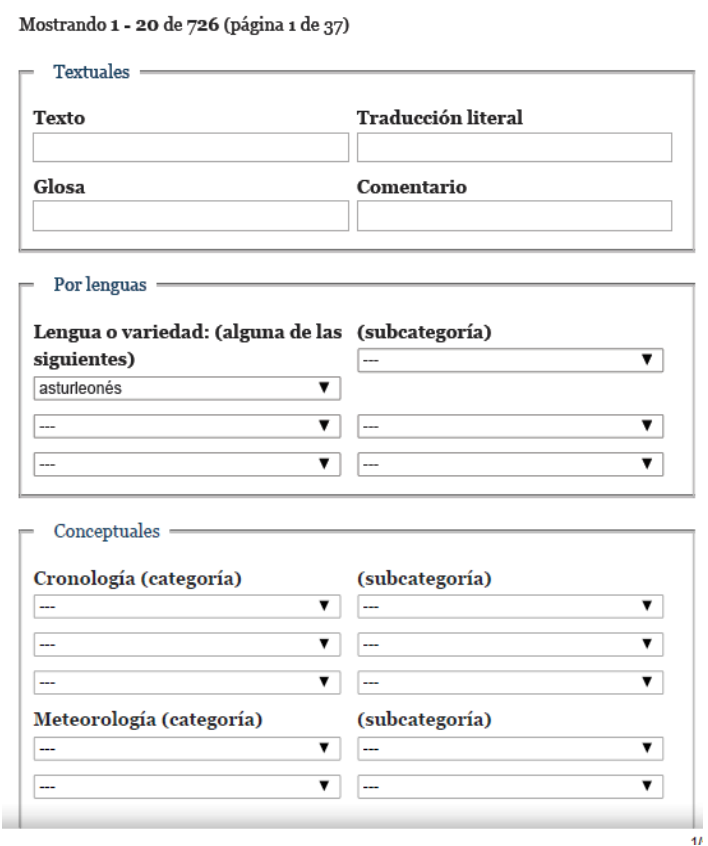

Figura 1

\section{2. ¿QUÉ ES UN REFRÁN METEOROLÓGICO?}

Se suele definir los refranes como afirmaciones concisas de verdades aparentes de uso corriente entre el pueblo o, de forma más elaborada, los refranes son sentencias breves, generalmente conocidas por el pueblo, que contienen sabiduría, verdades, enseñanzas y puntos de vista tradicionales en una forma metafórica fija y memorizable que se transmiten oralmente de generación en generación. Dentro de este gran grupo de la sabiduría proverbial, los refranes meteorológicos constituyen un subgénero. Su principal función es predecir el tiempo para que la gente pueda planificar las actividades diarias de la vida sin demasiadas incertidumbres o sorpresas climáticas. [...] (Mieder, 1996, p. 59)

La definición de refrán varía de unos diccionarios a otros. Según el 
DALLA (s.v. refrán, el): «Frase fecha [que contién una enseñanza, un conseyu]»; $\mathrm{y}$ este mismo diccionario define meteorolóxicu, $-a$, $-o$ como «De la meteoroloxía. 2 Del meteoru». El DLE, diccionario normativo del español, es mucho más escueto para su refrán: «Dicho agudo y sentencioso de uso común»; y define meteorológico, $-a$ como «Perteneciente o relativo a la meteorología o a los meteoros». Así pues, lo «meteorológico" vale para la meteorología como 'ciencia que estudia los fenómenos atmosféricos', pero también para los meteoros o 'fenómenos atmosféricos estudiados por la meteorología'. Y es que el adjetivo compuesto meteorológico, con su segunda parte basada en logos $\rightarrow$ logikos (como filológico), se aplica tanto al fenómeno estudiado como a la ciencia que lo estudia. De haberse producido una disociación semejante a la de climático ('relativo al clima') y climatológico ('relativo a la climatología o ciencia que se ocupa del clima'), tendríamos el uso asimismo disociado de meteórico (propio de los meteoros) y meteorológico (relativo a la meteorología, ciencia). Pero el uso común de meteórico, creo, se relaciona con la piedra meteórica (DLE, s. v. piedra), con el meteorito y su velocidad «meteórica». ${ }^{8}$

En cualquier caso, si casamos el sustantivo refrán con el adjetivo meteorológico, tenemos que el sintagma nominal refrán meteorológico vale, a efectos prácticos, como paremia ${ }^{9}$ con implicación meteorológica, atmosférica, climática, tanto si es referida a espacios de tiempo (meses, fechas, etc.) como si no presenta referencia cronológica explícita.

\section{FUENTES PRINCIPALES EN EL ÁMBITO ASTURIANOLEONÉS}

La base de datos de ParemioRom ha bebido de 190 fuentes escritas, que abrazan el último siglo y medio. En su día se contempló la viabilidad de realizar encuestas orales selectivas, pero la tentativa no pasó de algunas prospecciones en el ámbito del catalán y del aragonés.

En el del asturianoleonés, cuatro han sido las fuentes principales: (1) el Refranero asturiano de Luciano Castañón (1962a), aun si presenta, como el resto de obras de este autor, abundantes materiales en castellano o bien castellanizados, además de ofrecer opciones gráficas prenormativas y acaso objetables; (2) el Diccionario General de la Lengua Asturiana (DGLA) de Xosé

\footnotetext{
${ }^{8}$ Hoy mismo (un lunes de diciembre de 2019) he oído algo sobre la carrera meteórica de la cantante Rosalía.

9 Paremia es un grecismo, hiperónimo de registro culto y científico del que se sirve la paremiología, la ciencia paremiológica. Paremia es el nombre de una revista de referencia en el mundo de la paremiología ( $<$ https://cvc.cervantes.es/lengua/paremia/ $>$ ); sustantivo que el DLE define mediante una serie de hipónimos («Refrán, proverbio, adagio, sentencia»). Y no me entretendré en los múltiples desvelos que ha suscitado la diversa tipología del concepto 'paremia', pues no afecta al propósito de mi trabajo.
} 
Lluis García Arias (2002-2004), que tras su publicación en papel se volcó a una versión en línea; ${ }^{10}$ ni que decir tiene que los numerosos refranes para los que el filólogo tebergano bebe de Luciano Castañón se presentan conforme a la grafía normativa de la Academia de la Llingua Asturiana; (3) Ana Ma Cano González (1989), Notas de Folklor Somedán; es un opúsculo en que la autora reúne datos parémicos y folclorísticos de su Somiedu natal; (4) de F. Javier Rúa Aller (2014), Refranes del tiempo en León, se han extraído datos para 181 fichas del ámbito leonés.

En cambio, no se utilizó el libro de Xulio Viejo (2012), Paremias populares asturianas; entre otras razones, porque, a pesar de la muy nutrida y documentada recopilación del autor, venía a redundar sobre los paremiotipos que ya teníamos registrados en una fase avanzada de ParemioRom.

\section{Muestra ASturianoleonesa en SU CONTEXto RomanCE}

El objetivo básico de este artículo consiste en contextualizar la muestra de refranes meteorológicos del asturianoleonés en su marco romance, en ver el alcance de los distintos patrones parémicos en otras variedades hermanas de la Romania; pero igualmente en ver lo específico, lo singular, del romance asturianoleonés.

Indico en cada epígrafe los elementos nucleares de cada paremiotipo, su estructura básica. En cuanto a los refranes, son transcritos tal como figuran en las fichas de ParemioRom, que los toma literalmente de los correspondientes originales. Estos se consignan en la presente muestra solo de manera ocasional, para mejor ilustrar aspectos concretos de ciertos refranes. En las fichas de ParemioRom pueden consultarse tanto esas fuentes de referencia como otros detalles: así, pasajes citados de las diferentes obras y comentarios adicionales por parte del equipo investigador. En el caso de los refranes de la muestra localizados en Asturias, o sea asturianos sensu stricto, que son la mayoría, no se indica adscripción local; sí, en cambio, cuando proceden de León (con la abreviatura casi homónima de leon. = leonés). Por otra parte, el resto de testimonios romances se identifica mediante las abreviaturas correspondientes, ${ }^{11}$ y se dispone mediante una ordenación aproximativa de oeste a este. En cuanto a la transcripción textual, se indica ocasionalmente el

\footnotetext{
${ }^{10}$ https://mas.lne.es/diccionario/. Bendición de diccionario, equiparable en algunos aspectos, como la incorporación de fraseologismos y paremias, al DCVB, tal como señalé en mi recensión del DGLA (Gargallo, 2006, pp. 382-386) y reiteré en mi contribución al homenaje dispensado por la Academia de la Llingua Asturiana al prohombre de Teberga (Gargallo, 2010, p. 177).

${ }^{11}$ A saber: arag. $=$ aragonés; cast. $=$ castellano; cat. $=$ catalán; fr. = francés; gall. = gallego; it. = italiano; occ. $=$ occitano; piam. $=$ piamontés; port. $=$ portugués, roman. $=$ romanesco.
} 
salto de línea original con [/], y se añade coma entre corchetes para marcar la cesura de algunos refranes.

\subsection{Altura y proyección del sol. Xineru / Febreru $\rightarrow$ sol $\rightarrow$ regueru (Candelera $\rightarrow$ reguera)}

Tierra quebrada y de clima riguroso, cuando el sol anda bajo por el horizonte y no entra durante semanas o incluso meses en lugares umbríos, su gente celebra a través de refranes el regreso del sol, que es anuncio de primavera. El tipo léxico regueru (4.1.1. y 4.1.2.), con su femenino reguera (4.1.3.), se amolda a la rima con los nombres de xineru y febreru, sea a través de la mención genérica del mes, de su mitad (A mediaos de Febreru) o de una fecha tan señalada como San Antonio abad, San Antón de enero. Sobre la estructura básica que indican los epígrafes correspondientes, puede haber extensiones del refrán que enriquecen o matizan el contenido anterior (cinco de los nueve presentan algún tipo de añadido).

\subsubsection{Xineru / regueru}

- El día San Antón de Xineru ha de dar el sol en toos los regueros; ha de dar, pero no ha calentar.

- En Santo Antón de Xineiro entra 'l sol en toos los regueiros, menos en el de Rocabo, que no entra ' $n$ tol año.

- En Xineru entra'l sol en toos los regueros; ye refrán, pero non yé verdaderu.

- Nel mes de Xineru entra'l sol en cada regueru.

4.1.2. Febreru / regueru

- A mediaos de Febreru entra'l sol en cualquier regueru.

- En Febreru entra'l sol en cualquier regueru, menos en Enu.

- En Febreru entra'l sol en cualquier regueru; el últimu día[,] que no el primeru.

- En febreru, entra'l sol en cada regueru.

\subsubsection{Candelera / reguera}

El DGLA (s. v. candelera, la) localiza en Llanes El día de la Candelera entra el sol en cualquier reguera; Castañón (1963, p. 260), en El Mazucu (Llanes), esta otra variante con Candeleras en plural: El día de las Candeleras entre'l sol en todas las regueras.

Dicho refrán con sus Candelera, sol y reguera me suscita otro de Cantabria, que el ALECant (mapa 890: Candelaria) localiza en Arredondo 
(punto de encuesta $\mathrm{S} 405$ ), y que nos indica cómo en esta fecha no menos señalada del 2 de febrero (cfr. 6.1.) se festeja también el sol recobrado: En las candelas entra el sol por todas las callejuelas.

Muchos otros refranes romances retratan el sol que vuelve a entrar por las umbrías, como el castellano San Matías, ya entra el sol por las umbrías; paremiotipo representado asimismo en el Alto Aragón y en el catalán meridional, valenciano incluido (Correas y Gargallo, 2002).

\subsubsection{Confrontación romance}

Los refranes del asturianoleonés aducidos responden a un paremiotipo bien característico, que halla ecos en portugués, en gallego y en castellano (¿regional?):

- Port. Em Fevereiro, entra o sol em cada regueiro.

- Gall. En xaneiro entra o sol en cualquiera regueiro, pero é no cima que non é no primeiro (ALGa, p. 462, núm. de refrán 32; en el punto de encuesta O.8, Larouco, Ourense).

- Cast. En el mes de Enero [/] Entra el sol en cada reguero (Rodríguez Marín, 1896, p. 39). Me pregunto si es de uso regional, o acaso un calco paremiográfico a partir de moldes gallegos o asturianoleoneses, pues no me consta su empleo, de fuente oral, en el castellano de otros lugares de la península.

\subsection{Meses}

\subsubsection{Enero $\rightarrow$ otero...}

Semejante atracción de rima se da, ahora entre los representantes asturianos de JANUARIU (a través del latín vulgar JENUARIU) y ALTARIU, en la primera parte del refrán En Xeneiro súbete al outeiro, si ves verdexar ponte a bailar, si ves terreisar, ponte a tchorar; que se completa con dos formulaciones condicionales en que el verdor de la tierra invita al contento, mientras que el terrear del entorno, al llanto. Lo recoge Castañón (1962a, p. 159) en Santa María del Puertu (Somiedu), con esta glosa: «Si ves verdear, alégrate, buen año; si ves la tierra quemada de la helada, llorar, por mal año». Y el mismo autor lo confronta con el gallego: «Pero en Galicia $[\ldots] »$.

En Galicia y en Portugal, en gallego y en portugués, se documenta una estructura idéntica pero con expectativas inversas: gall. En xaneiro ponte no outeiro; se ves verdegar, ponte a chorar; e se ves secar, ponte a cantar; port. Em Janeiro põe-te no outeiro; se vires verdejar, põe-te a chorar; se vires terrear, põe-te a cantar.

En el caso de cierto registro en castellano, da que pensar que se trate 
asimismo de un calco paremiográfico a partir de alguna de las variedades iberorromances occidentales, a no ser que se inscriba en el castellano regional occidental, o incluso de áreas bilingües: En enero ponte en el otero, y si vieres verdeguear ponte a llorar, y si vieres terreguear ponte a cantar (Martínez Kleiser, 1945, p. 180). Todo cabe en un repertorio acumulativo y sin localizaciones explícitas como este.

\subsubsection{Febrero $\rightarrow$ miraero...}

El paremiotipo representado por A mediaos de Febrero asómate al miraero; si él rí, tú llora, y si él llora, tú rí convida asimismo a subir y a mirar el entorno; en su caso, mediante un complemento de predicción inversa basada en el reír o llorar metafóricos de la tierra que, ahora sí, recuerda a los inmediatos testimonios anteriores (4.2.1.) de gallego y portugués. Una variante de la segunda parte se ofrece en este otro testimonio: A mediaos de Febrero ponte'nel miraero; si lo ves blanco cantarás, si lo ves verde llorarás. No encuentro homólogos romances para dicho paremiotipo.

\subsubsection{Marzo marceador, Marzo marzán}

Tal como señalé en Gargallo (2003), son muchos los refranes romances en que una primera parte invoca a Marzo ([sic] humanizado, con mayúscula inicial de nombre propio) mediante el continuador de MARTIUS seguido inmediatamente de una duplicación denominativa y derivada ad hoc, como en el marceador y el marzán de los dos refranes que transcribo más abajo. El correspondiente paremiotipo principal, que empieza con una invocación duplicada a Marzo, presenta un subparemiotipo en que se contrasta el tiempo de la mañana con el de la tarde conforme a la variabilidad característica del mes:

- Marzo marceador, pe la mañana de sol rayaes, pela tarde turbonaes.

- Marzo marzán, por la mañana cara de risa y por la tarde de can.

Diversos testimonios romances responden a un patrón afín: gall. Marzo marcián, pola mañá cariña de rosa e pola tarde cara de can; port. Março, marçagão, [/] Pela manhã rosto de cão [/] À tarde sol de verão; cast. Marzo marcero, por la mañana rostro de perro, por la tarde valiente mancebo; Marzo, marcero: por la mañana cara de perro[,] y por la tarde todo un caballero; cat. Març, marçó, | al matí cara de gos $\mid i$ al vespre galant $i$ minyó.

\subsubsection{Mayo / sayo}

Mayo atrae la rima consonante con sayo en diversas variantes de un tipo parémico iberorromance en que se previene sobre el aligerarse de ropa, aún en ese mes, ante la eventualidad de fríos tardíos. Sayo, al parecer extraído del femenino saya (DCECH, s.v.), presenta en castellano, según el DLE, una acepción de uso pretérito ( $« 1 . \mathrm{m}$. Prenda de vestir holgada y sin botones que cubría el 
cuerpo hasta la rodilla»), así como otra moderna y genérica, que el diccionario académico marca como coloquial: la de 'vestido' («prendas con que se cubre el cuerpo»). El DALLA, por su parte, reúne hasta nueve acepciones bajo sayu. En cualquier caso, resulta significativo que dicho tipo léxico, que reviste valores relativos al abrigo en iberorromance, haya propiciado la rima con los herederos de MAJUS y, con ello, el paremiotipo correspondiente: en castellano y en asturianoleonés, en gallego y portugués, y en aragonés también. No se da, sin embargo, en catalán, que ni conoce un homólogo etimológico de sayo ni, lógicamente, da pie a la rima. ${ }^{12}$ Vuelvo sobre la atracción de rima entre mayo y sayo a propósito del tipo jocoso del 40 de mayo (cfr. 4.3.2.).

\subsubsection{Asturianoleonés}

- Anque veas el sol de Mayo, non quites el sayo.

- Asfoutu del sol de Mayo no te vayas sin el sayu; asfoutu del mes d'Abril no vayas sin qué cubrir. Presenta una segunda parte con un consejo de abrigo adicional para el mes previo.

- En enfouto del mes de Mayu, el pastor non dexe'n casa la capa nin el sayu.

- En ficias del mes de Mayo, nun deixes el sayo.

- En Mayu non pierdas el sayu.

\subsubsection{Confrontación romance}

Gall. Garda o teu saio para maio; port. Guarda o melhor saio para Maio. Cast. En mayo, no dejes el sayo, por si en vez de derechero viene de soslayo. Guarda el sayo, para mayo. Arag. En mayo / quitate el sayo; / en abril / no te'n quites fil; su segunda parte recuerda el prevenir sobre abril del segundo refrán asturianoleonés de la serie anterior. Nótese, por otra parte, que en este refrán, localizado por Gomis (1998, p. 161, n. 25) en la ribera del Ésera, la forma fil, que posibilita la rima gracias a la pérdida de la vocal final (de FILU), apunta hacia el catalán en esa zona fronteriza de habla aragonesa ribagorzana.

Otro es el caso del sardo: In su mese de Abrile non tocches unu pile, in su mese de Maju non tocches su saju; ¿quizá préstamo parémico del castellano? Sorprende, por una parte, la forma pile 'pelo', que debiera ser pilu según el tipo romance sardo, y que muy probablemente viene forzada por la rima con Abrile. Por otra parte, la interpretación que una de las fuentes de referencia ofrece del refrán converge con la del susodicho paremiotipo iberorromance: «Vuol dire, nell'aprile e nel maggio non alleggerir gli abiti [...] Spagn.[uolo] En el majo non dexas el sajo (da SAGUM)» (Spano, 1997 [1871], p. 226).

\footnotetext{
${ }^{12}$ Traté sobre este y otros tipos de refranes romances con consejos de abrigo en Gargallo (2007a).
} 


\subsubsection{Mayo pardo y junio claro}

El contraste entre un mes de mayo pardo, oscuro, acaso lluvioso, y un mes de junio claro augura buena cosecha, según cierto paremiotipo documentado en el occidente peninsular:

Mayo pardu y Xunio claru, siñal de bon añu; Moyu pordu y Xunu cloru[,] habrá pan y vino fortu; este último, localizado por Castañón (1962b, p. 410) en Sotu y Agues (Sobrescobiu); y en otra obra (Castañón, 1963, p. 271), en Sotu (Sobrescobiu), con la grafía xloru (posible errata). Nótese la metafonía de tipo velar $(a>o)$. El DGLA (s.v. mayu, el) remite a Luciano Castañón, ofrece la localización de Sobrescobiu y transcribe xunu con inicial minúscula. Junto a estos cabe mencionar asimismo la variante Mayu brumosu y Xunio claru, saquen la cosecha de tol añu.

Los bueyes y el carro como expresión de la abundancia caracterizan estos dos homólogos del gallego y del portugués: Maio pardo e san Juan claro valen máis có boi e o carro; Maio pardo e Junho claro podem mais que os bois e o carro.

Por otra parte, se dan retahílas de meses con la culminación de un mayo pardo y un San Juan claro; y San Juan parece referirse a 'junio', al menos en estos primeros ejemplos del gallego: Con xaneiro xeadeiro, febreiro nevareiro, marzo sollarzo, abril mollado, maio pardo, san Juan claro valen máis que tus mulas y tu carro; Frebeiro greleiro, marzo airoso, abril chuviñoso, maio pardo e san Xoán claro son as chaves de todo o ano; Marzo airoso, abril chuvioso, maio pardo e san Juan claro valen máis que as mulas e o carro. En castellano: Un enero helado, un febrero amoroso, un marzo airoso, un abril lluvioso, un mayo pardo, un San Juan claro, ya valen más que la mula y su carro; Un febrero chapucero, un marzo airoso, un abril lluvioso, un mayo pardo, un San Juan claro, valen mucho más que tu mula y tu carro.

Se dan también otras combinaciones de meses; por ejemplo, con la anticipación de «Abril lluvioso»: gall. Abril chuvoso, [/] Maio pardo e ventoso [/] Faz o ano formoso; cast. Abril lluvioso, Mayo pardo y Junio claro, valen más que los bueyes y el carro.

En cualquier caso, el paremiotipo antedicho con mayo pardo y junio claro, así como las retahílas de meses previas que los incluyen, se documentan de manera exclusiva en el espacio iberorromance; $y$, en cuanto a los ejemplos del castellano, no es descartable que sean eco del occidente peninsular (¿castellano regional?, ¿calcos paremiográficos?).

4.2.6. Lluvia de agosto $\rightarrow$ miel y mosto

Ambos sintagmas constituyen el sostén de las dos partes que componen cierto paremiotipo de alcance general en el continuo territorial románico, al que se adscribe nuestro testimonio asturiano: Si llueve 'n Agostu, lloverá miel y 
güen mostu.

Selecciono algunos de los posibles homólogos romances: gall. Cando chove por agosto, chove mel e mosto; Chuvia en aghosto, mel e mosto; port. Quando chove em Agosto, chove mel e mosto; cast. Cuando llueve en agosto, llueve miel y mosto; occ. Quoan plau en Aoust, [/] Que plau méu e moust; romanche (retorrománico de Suiza) Cur il plova nel Avust [/] il plova mel e bun most; fr. La pluie du mois d'août [/] c'est du miel et du moût; Quand il pleut en août, [/] Il pleut miel et bon moût; it. Quando piove d'agosto, piove miele e piove mosto; Se piove d'agosto, piove olio, miele e mosto.

\subsubsection{Septiembre $\rightarrow$ puentes y fuentes}

Mes de extremos meteorológicos, septiembre puede ver lluvia a cántaros o alternativamente sequía. La concreción de uno y otro estados mediante (1) puentes que se lleva el agua de lluvia desmedida, o bien (2) fuentes secas, descansa en sendos tipos léxicos, herederos de PŎNTES y FŎNTES, que facilitan la rima por toda la Romania parémica continua. Y en dicho continuum se inscribe asimismo el asturiano: Setiembre, o lleva los puentes o seca les fuentes. Además, el tiempo de septiembre puede singularizarse en fechas concretas del mes: Per San Antolín o se sequen les fuentes o lleva'l rio les puentes [San Antolín, 2 de septiembre]; Por San Miguel o seca las fontes o lleva las pontes [San Miguel, 29 de septiembre].

Por otra parte, los numerosos homólogos romances que responden a este paremiotipo presentan tanto el orden señalado (fuentes / puentes) como el inverso (puentes / fuentes); siempre al dictado de la rima consonante:

- Gall. No setembre ou secan as fontes ou levan as pontes; Setembro ou leva as pontes ou seca as fontes.

- Port. Setembro, ou seca as fontes ou leva as pontes.

- Cast. Septiembre, o lleva los puentes o seca las fuentes; Septiembre, o seca las fuentes o se lleva los puentes.

- Arag. Setiembre, u seca as fuentes [/] u se lleba os puentes.

- Cat. El setembre s'enduu els ponts o eixuga les fonts.

- Occ. Setèmbre emporto li pont [/] $O$ bèn agoto li font; occ. aranés Seteme o sheque es hònts [/] o s'empòrte es pònts.

- Fr. Septembre emporte les ponts [/] Ou tarit les fonts.

- It. Di settembre [/] o porta via i ponti [/] o secca le fonti.

4.2.8. Luna de octubre

La luna septembrina, según creencia popular de muchos países 
romances, prefigura el tiempo de siete lunas o meses por venir (así, en catalán La lluna setembrina $\mid$ set llunes endevina; o en italiano Alla luna settembrina [/] sette lune se ne inchina), en lo que pudiera haber influido la percepción del 'siete' en el nombre del mes, conforme a su etimología (SEPTEMBER). Y acaso esta predicción septenaria desde septiembre haya servido de pauta para las siete lunas o meses que también prefigura la luna de octubre. ${ }^{13} \mathrm{Ni}$ que decir tiene que el crédito científico que merece tal tipo de previsión es nulo; poco más o menos, como la incidencia de las doce uvas de Nochebuena de cara a los doce meses del año entrante, que, curiosamente, tantas ilusiones suscitan.

- La lluna d'Otubre, siete llunes cubre.

- Si nieva 'na luna d'Octubre, siete meses la cubre.

Hallo correspondencias en otras variedades peninsulares, como el gallego A luna de otubre [sic], sete cubre, y el castellano: La luna de octubre, siete lunas cubre; La luna de octubre, siete lunas cubre; y si llueve, nueve.

En el dominio lingüístico catalán no es un paremiotipo general: Lluna d'octubre, | set ne descubre es refrán tomado por Sanchis (1951, p. 129) de fuente tortosina, como en el DCVB (s. v. lluna y s. v. octubre). Manent (1997, p. 43) localiza en Maella (provincia de Zaragoza): Lluna d'octubre siete en descubre (nótese el castellanismo siete en esta variedad periférica del dominio lingüístico). Por otra parte, la forma verbal descubre (por la normativa descobreix) es seguramente otro castellanismo que la rima facilita.

Asimismo en catalán: En octubre la llunà, | set atres cobrirà; recogido por Sanchis (1951, p. 129) de fuente valenciana, de la que también lo extrae el DCVB (s. v. octubre), pero con distinta preposición al inicio y sin marca de cesura: D'octubre la llunà, set atres cobrirà. Nótense los dialectalismos llunà (por llunada) y atres (por altres).

Y todavía en catalán: Octubre el vanitós, | cobrix set llunes enganyós; en Sanchis (1951, p. 129), que lo registra de fuente valenciana (nótese el dialectalismo cobrix, propio del catalán occidental; en catalán oriental, cobreix). En Amades (1951, p. 994), uno de tantos repertorios acumulativos del mundo de la paremiografía, se recoge sin localización: L'octubre vanitós cobreix set llunes enganyós.

4.2.9. Noviembre truena...

Nuevamente la fuerza de la rima, ahora entre los representantes románicos de TRŎNAT y BŎNA, guía la forja de un refrán: Cuando ' $n$ Noviembre trona, la coliecha siguiente será bona. El paremiotipo se halla representado en otros romances: cast. Si en noviembre oyes que truena, la cosecha será buena;

${ }^{13}$ Sobre refranes con luna de octubre, véase Gargallo (2007b, p. 121). 
cat. Si pel novembre trona, | la collita serà bona; fr. Si en novembre il tonne, [/] L'année sera bonne; it. Se di novembre tuona, l'annata sarà buona.

4.2.10. Diciembre mojado $\rightarrow$ enero helado

En este otro la correlación de la lluvia en diciembre con el hielo de enero halla reflejos de cabo a cabo de la península ibérica; en asturiano: Si ye Diciembre moyau, será Xinero xelau; gall. Nadal mollado e xaneiro ben xeado $[$ Nadal $=$ 'diciembre']; port. Dezembro molhado, Janeiro geado; arag. Diziembre mojau, enero bien chelau (testimonio de Palacios y Baos, 2010, 168; presenta el castellanismo mojau); cat. Desembre mullat, | $i$ gener ben gelat.

\subsection{Fechas fijas}

\subsubsection{La Candelaria, 2 de febrero. La Candelera llora/chora $\rightarrow$ iviernu fora}

Cuarenta días después del de Navidad ( 25 de diciembre), en un recuento inclusivo, el de la Candelaria constituye un hito de referencia en el corazón del invierno. La «Virgen de las Candelas», con el simbolismo de su luz, alumbra en una mayoría de lenguas romances nombres derivados del sustantivo CANDELA. Parece ser que una reinterpretación de la base (FESTA) CANDELARUM 'fiesta de las candelas' habría dado lugar a lo que solo en apariencia son derivados en -ARIA, como la Candelaria de terminación culta y la Candelera / Candeleira que presenta tratamiento popular del sufijo, entre otros continuadores romances (Tagliavini, 1963, pp. 195-199; DCECH, s.v. candela).

Pues bien, el soporte simbólico y cronológico de la Candelaria, y la metáfora de su llorar (llover), constituyen la primera parte de cierto paremiotipo iberorromance que augura de diversos modos una predicción inversa (buen tiempo) para el resto del invierno; predicción expresada mediante una segunda parte que acomoda la rima de fora -así, sin diptongación- al verbo precedente (llora/chora). Es posible, además, un complemento de predicción nuevamente inversa, ahora con el reír (sol, buen tiempo) de la Candelaria y el consiguiente recrudecerse del invierno. Y hasta se dan expansiones del paremiotipo que constatan la persistencia del tiempo de invierno tanto si la Candelera ríe como si llora. De las 75 fichas de refranes en ParemioRom extraigo los siguientes testimonios asturianoleoneses: ${ }^{14}$

- Si a Candeleira chora, el invierno xa vay fora; si a Candeleira rí, el invierno ta por vir; Si la Candelera llora, el ivierno fora; si la Candelera rí, el ivierno por vinir.

- Cuando la Candelera chora, el invierno vai fuara, que chore que deixe de chorar, la mitá del invierno ta por andar; leon. Si la Candelaria

${ }^{14} \mathrm{Y}$ remito para otros detalles sobre este paremiotipo a lo expuesto en Gargallo (2004, pp. 117 121). 
chora, l'iviernu está fora; si rii, por venir; que chore, que deixe de chorar, la mitá pasáu, la mitá por pasar; leon. Si la Candelaria xora I l'uviernu ya está fuora. / Que chore que deije de chorare, / l'uviernu ta por chegare; Si la Candelera llora, el iviernu ya ta fora; si nin llora nin dexa llorar, el iviernu ta sin pasar; Si la Candelera llora, el iviernu ya va fora, pero si non llora, nin dientro nin fora.

El siguiente invierte el orden más habitual del llorar y el reír de la Candelaria: leon. Si la Candelaria rire, / l'hibiernu por venire, / Si la Candelera llora, / l'hibiernu fora, / que llore ya que cante, / l'hibiernu atrás ya alantre.

Y este otro, localizado por Castañón (1962a, p. 272) en Quirós, formula negativamente el llorar del día, pero hace prevalecer la rima habitual entre chora y fora: Si la Candelera non chora, el invierno fora.

Confrontación romance selecta: gall. Cando a Candelaria chora, medio inverno vai fóra; port. Em Fevereiro, se a Candelária chora, está o Inverno fora. Se a Candelária rir, está o Inverno p'ra vir; Se a Senhora da Luz está a chorar[,] está o Inverno a acabar; se está a rir, está o Inverno para vir (con esta designación portuguesa para la Virgen de las Candelas, hecha de $L u z$ ); cast. Cuando la Candelaria plora, / invierno fora; Cuando la candelera plora, el invierno flora (ALEANR, mapa 1148, punto de encuesta Na 206, Roncal); arag. Cuando a Candelera plora [/] l'imbierno ya está fora; Si la Candelera plora, l'imbierno ya ye fora; benasqués (geotipo romance específico entre el aragonés y el catalán) Si la Candelera plora, [/] l'hivern ja é fora. [/] Plourà o no plourà, [/] l'hivern s'acabarà; cat. Si la Candelera plora / l'hivern fora... Tal es su popularidad en catalán que, si se le propone ese inicio a un hablante genuino, es bien previsible que continúe con variantes como l'hivern és viu, torna-te'n al niu, entre otras.

\subsubsection{Hasta el 40 de mayo...}

Se diría una deriva del paremiotipo que aconseja no aligerarse de abrigo (no quitarse el sayo) aún por mayo (cfr. 4.2.4.). La invención del 40 de mayo obedece, no sin cierta jocosidad, al peso simbólico de la cuarentena: en el calendario, en nuestra civilización, en la vida (Gargallo, 2018a, 2018b). Se da asimismo en iberorromance (no en catalán, en que no hay sayo que valga para la rima).

Junto al asturiano (Hasta'l cuarenta de Mayu non te quites el sayu), aporto una selección de testimonios iberorromances, algunos de los cuales presentan ampliaciones parémicas en relación a junio o bien a una eventual lluvia: gall. Ata o corenta de maio, non te quites o saio; Hastra o corenta de maio non te quites o saio[,] e, si eres de Lugo, hastra o trinta de xunio (este último, recogido por Vázquez Saco, 2003, p. 133); cast. Hasta el cuarenta de 
Mayo [/] No te quites el sayo; [/] Y si Junio es ruin, [/] Hasta su fin; arag. Basta'l cuarenta de mayo no te saques el sayo, y si beis que ba plever[,] tornalo a meter.

Párrafo aparte merece el testimonio alpino de la variedad lombarda de Poschiavo, en los Grisones: Fin al quarànta da macc nu tra fó $i$ strasc ['Hasta el cuarenta de mayo no te quites el abrigo']. Posiblemente a muchos hispanohablantes les sorprendiera hallar en la Suiza alpina una réplica del conocido refrán castellano Hasta el cuarenta de mayo...

4.3.3. Agua (orbayu) de San Juan...

Según el refranero, por San Juan (24 de junio) la lluvia no es propicia para la cosecha. A una primera parte del refrán en que se expresa agua (o bien orbayu) en ese día, sigue el mal augurio de vino y pan, con la rima apropiada:

- Agua de por San Xhuan quita vinu y non da pan; atribuida por el DGLA (s.v. agua, $l^{\prime}$ ) a Junquera Huergo; El agua en San Juan tolle el viño y non fai pan (Occidente, según Castañón, 1962a, p. 121); El agua por San Juan quita vino y non da pan; L'augua por San Xuan quita vinu ya nun da pan.

- El orbayu per San Xuan quita vino y non da pan; El orbayu per San Xuan quita yerba y non da pan.

Confrontación romance: gall. Agua de San Joan, tolle viño e non dá pan; port. Água de S. João tira vinho e não dá pão; cast. Agua por San Juan [/] Quita vino y no da pan; arag. Agua pa' San Juan quita vino y no da pan; occ. Li plueio de sant Jan [/] N'aduson ni vin ni pan; fr. L'eau de la Saint-Jean ôte le vin [/] Et ne donne pas de pain.

\subsubsection{Todos los Santos y San Andrés, y la nieve}

Todos los Santos, al principio de noviembre, busca la rima con una nieve aún por los altos: Per Toos los Santos, la nieve pe los altos. Al cabo del mes, San Andrés (30 de noviembre) la encuentra a la puerta de casa, en esta extensión del paremiotipo anterior: El día Toos los Santos la nieve pe los altos, y per San Andrés, a la puerta lo tendréis.

En otros testimonios de la península, referidos a ambos cabos de noviembre, San Andrés quiere la rima con 'pies': gall. Nos Santos, neve nos altos; en San Andrés, neve nos pés; cast. Por los Santos, la nieve en los altos; por San Andrés, la nieve en los pies; arag. Pa' T'os Santos nieve por os altos; pa'San Andrés nieve por os pies; cat. Per Tots Sants la neu pels alts, i per Sant Andreu la neu al peu. Este último, recopilado por Amades (1951, p. 999), bien pudiera ser un calco paremiográfico del castellano. 


\subsubsection{El veranín de San Martín}

En el corazón del otoño, el rastro de la veneración a San Martín de Tours (11 de noviembre) ha sembrado por la Europa occidental innumerables elementos de cultura popular, entre los cuales la creencia en un veranillo, un verano tardío atribuido al episodio hagiográfico según el cual el santo quiso partir su capa con un mendigo que padecía de frío, y el buen Dios dispuso traer un breve verano para alivio de mendigo y santo (Bibliotheca Sanctorum, s.v. Martino).

Aun con la circunspección que va imponiendo en los últimos tiempos la conciencia del cambio climático, persiste por buena parte de la Romania la creencia en un "veranillo de San Martín», que se inscribe en un contexto europeo de manifestaciones afines de cultura popular, entre lo cristiano y lo pagano (Gargallo y Ruiz-Zorrilla, 2012).

El tipo léxico romance «veranillo/verano de San Martín» se halla bien representado en la base de ParemioRom: gall. vranciño de San Martiño, port. verão de San Martinho, cast. veranillo de San Martín, cat. estiuet de Sant Martí, fr. été de la Saint-Martin, it. estate di San Martino. Y participa en refranes como el asturiano El veranín de Samartín tien que venir, que expresa de manera sencilla la expectativa de ese verano otoñal.

\subsubsection{Navidad (y Pascua)}

No son pocas, en el calendario romance de refranes (Correas y Gargallo, 2003), las que llamo «predicciones inversas», como la ya señalada (4.3.1.) de una Candelaria «llorosa» o lluviosa anunciadora de buen tiempo, y la de otra risueña que con buen tiempo augura malo. Se trata de una filosofía compensatoria muy humana, que también se da entre dos de las festividades más señaladas del año, Navidad (25 de diciembre) y Pascua de Resurrección; fecha movible, esta, que llega con la primera luna llena de la primavera. Una de las posibles manifestaciones de tal predicción inversa confronta el sol navideño con una Pascua fría, al amor de la leña o el tizón: El que per Navidá s'asoleya, per Pascua quema la lleña; Les Navidaes al sol y les Pascues al tizón; Navidades al sol[,] pascuas al tizón.

Para el primero de los tres refranes asturianos sugiero estos homólogos occitanos: Quau pèr Calèndo se souleio, [/] Pèr Pasco brulo sa ligno; Qui pèr Nadau s'assoulelho, [/] Pèr Pascos s'estourrelho.

Para los otros dos, con tizón, los siguientes: cast. Por Navidad, sol; / por Pascua, tizón; occ. Nadàu au sou, Pasques s'ou tisoû; piam. A Natal 'l sulet, a Pasqua 'l tisunet; roman. Natale cor sole, Pasqua cor tizzone; abrucés Natale cójjo sòlo, Pasqua cójjo tizzóno. 


\subsection{Meteoros diversos}

\subsubsection{Arreboles}

La búsqueda temática en ParemioRom de cielo $>$ arreboles ${ }^{15}$ devuelve 412 fichas de refranes; 19 en asturianoleonés. Como en otros ámbitos romances, los arreboles se expresan mediante diversos -y sugerentes- tipos léxicos (bermeyón / bermellón, bermeyura; cielu colorau; encarnau; rubién), y son precisamente los que guían la clasificación de más abajo. Implican el saliente y el poniente, y por extensión, la mañana y la tarde. Cosa lógica, pues los arreboles necesitan del primer o del último sol del día.

4.4.1.1. Bermeyón / bermellón, bermeyura

- Bermeyón al saliente, l'agua de repente.

- Pa bon tiempu, bermellón al poniente y non al saliente.

- Pe la tardi bermellón, que pe la mañana non.

- Bermeyura de tarde, pa sol o p'aire; bermeyura de mañana, p’aire o p'agua.

4.4.1.2. (Cielu) coloráu / -ao

- Cielu colorao, suelu moyao.

- Si está colorau el cielo, aire ahora, agua lluego.

- Colorao al alba, o aire o agua; colorao a la cena, bon tiempu 'spera.

- Colorau colorón, a la terde, ca la mañana non.

- Colorao pa Pravia, bon día de sol mañana. Localizado en el Concejo de Villaviciosa por Luciano Castañón (1962a, p. 64). Cabe señalar el interés de los refranes que incluyen referencias geográficas, como en este caso, en que se singulariza la mirada de Villaviciosa hacia poniente (Pravia), en lo que no puede ser más que un cielo colorao del atardecer.

4.4.1.3. Encarnáu / -ao

- Encarnao pa Burón, sol a trompón, por la tarde sí, de mañana non; localizado en Cangas de Narcea por Castañón (1962a, p. 144), que glosa: «Burón: valle entre Asturias y Lugo». A dicho autor remite el DGLA (s. v. trompu, $\{e l\}$ ). Y este refrán mira también a poniente.

- Encarnau al alba, a la noche agua; encarnau al escurecer, agua al amanecer.

\footnotetext{
${ }^{15} C f r$. el DLE (s. v. arrebol): «1. m. poét. Color rojo, especialmente el de las nubes iluminadas por los rayos del sol o el del rostro».
} 
4.4.1.4. Rubién

- Rubién de cena buen día 'spera, si chovendo non se queda. Localizado por Castañón (1962a, p. 259) en Vil.laoril (Navia). El DGLA (s. v. rubién, \{el\}) localiza en Tox: Rubién de cena bon día espera, si chovendo non se queda. Halla continuidad en el tipo léxico roibén(s) del gallego, que se incluye en gran número de refranes de ParemioRom.

\subsubsection{Confrontación romance}

La designación más común de los arreboles en catalán es cel rogent. Y significativamente el diccionario académico (DIEC, s. v.) recoge bajo el adjetivo rogent, «Vermellós, s'aplica al cel», el refrán Cel rogent: pluja o vent, muy extendido por todo el dominio lingüístico. Da una idea de su popularidad el reconocimiento que obtuvo en la encuesta digital Els refranys més usuals de la llengua catalana (Fontana y Gargallo 2018, p. 41), así como el hecho de que sea uno de los preferidos en la información meteorológica de los distintos medios de comunicación en esta lengua.

De entre las designaciones correspondientes en castellano, destaco la muy «sanguínea» de vaca desollada; así, en los testimonios del ALeCMan (mapa 938, Arreboles del amanecer): Vaca desollada a saliente, agua a poniente (punto CU 204: Tragacete); Vaca esollá al saliente, suelta los bueyes $y$ vente (GU 410: Checa).

\subsubsection{Cielo aborregado y afines}

La búsqueda temática de cielo aborregado, una de las numerosas denominaciones para cierto tipo de nubes del género Cirrocumulus y Altocumulus, que recuerdan un rebaño de ovejas (Martín-Vide, 2011, pp. 253-254), ofrece 313 fichas en ParemioRom; y 14 en asturianoleonés. En los refranes de este ámbito romance predomina la designación de cielo empedrao (4.4.2.1.), pero hay otras como el cielu de llana que cierra el misceláneo punto 4.4.2.2. Y todos esos refranes coinciden en anunciar lluvia, en algunos casos con una indicación explícita de inmediatez ( $a$ les veinticuatros hores, al otru día a los dous días, a los tres días, antes de los tres dies; si no hoy, mañana). ${ }^{16}$

\subsubsection{Cielo empedrao}

Cielu empedrao, a les veinticuatro hores moyao; Cielu empedrao, agua 'n teyao; Cielu empedrao, al otru día suelu moyao; Cielu empedrao, suelu

\footnotetext{
${ }^{16}$ Martín-Vide (2011, p. 253) otorga solo cierta credibilidad a este tipo de refranes, tal como deja entender el epígrafe de Refranes que tienen algún fundamento, pero que no resisten la verificación estadística.
} 
moyao; Cielu empedráu[,] al otru día moyáu; Cielu empedriáu[,] a los tres días mocháu; Cielu empredao [sic], al otru día suelu moyao (Castañón, 1962a, p. 61); Cielo empedradín, agua pol camín.

\subsubsection{Otros}

- Cielo arbeau[,] suelo moyau; Cielu arbeyáu, agua nel teyáu.

- Cielo escalludo, vendoval seguro; Cielo escamudo, vendovaludo.

- Cielu enladrilláu, a los dous días mocháu (suelu mocháu).

- Cuando veas el cielu de llana, antes de los tres dies, agua; gall. Ceu de lan, chove mañán; cast. Cielo de lana, si no llueve hoy, lloverá mañana.

\subsubsection{Cercu de lluna y cercu de sol}

Hay refranes con cerco o halo de luna, y los hay con halo o cerco de sol; unos y otros, de predicción varia, de lluvia o buen tiempo. Y hay registros de refranes con cerco de luna y a la vez con cerco de sol, ${ }^{17}$ como el asturiano Cercu de lluna non hincha la llaguna; cercu de sol mueya al pastor; que confronta con estos otros: gall. O circo do sol molla o pastor, o da lúa ou molla ou enxuga; cast. Cerco de sol remoja al pastor; cerco de luna, o le moja o le enjuga (y múltiples variantes); arag. O rolde d'sol [/] moja a capa d'o pastor [/] y o d'a luna [/] le'n enchuga.

\subsubsection{Lluna echada, marinero en pie}

Maria-Reina Bastardas (2016) analiza distintas versiones romances del refrán aquí representado por el asturiano Lluna echada, marinero en pie (Castañón, 1962a, p. 197), que este otro autor glosa así:

Quizá porque entonces sube el pescado y se puede coger mejor. Pero más cierto porque cuando está acostada la luna, es decir con los picos p'arriba, barrunta mal tiempo y hay que estar prevenido. Son muy pocas las veces que se ve acostada. (ibidem)

Por su parte, el DGLA (s. v. lluna, la) transcribe marineru (con -u) y remite a Luciano Castañón. Es manifiesta la afinidad con el gall. Lúa deitada, mariñeiro a pe; port. Lua deitada, [/] Marinheiro em pé; incluso en el adjetivo deitada, del mismo origen ( $<$ JECTATA). Idéntica estructura presentan el cast. Luna acostada, marinero en pie; el cat. Lluna ajaguda, mariner dret; y el it. Luna coricata, marinaio alzato. Esa luna que pocas veces se ve acostada, luna incipiente del atardecer o menguante extrema del amanecer, se conoce en italiano popularmente como luna a barchetta, desde una mirada ciertamente marinera.

\footnotetext{
${ }^{17}$ Sobre refranes romances con sol y luna, véase Gargallo y Fontana (2018).
} 


\subsection{5. (Viento) nordeste}

La especificidad del (viento) nordeste, o nordés, en el noroeste peninsular se manifiesta a través de su presencia en un buen número de fichas de refranes en el ámbito asturianoleonés (10) y en el gallego (26). Transcribo aquellos con alguna confrontación gallega:

- Agues [sic] fríes y nordeste, la sardina «desaparece» (Castañón, 1962a, p. 61). En el DGLA: Agües fries y nordés, la sardina desapaez.

- Cuando de nordeste llueve, hasta les piedres mueve; cfr. gall. Cando con nordés chove, as pedras se moven; Cando d'o nordeste chove, [/] hasta as pedras move.

- Costa cargada, nordeste 'na playa; Montaña cargada, nordeste'na playa; Sierra cargada, nordeste en la playa (Castañón, 1962a, p. 270; en Puertu Veiga, Navia); cfr. gall. Serras cargadas, nordeste nas praias.

- El nordés que dura, de nueite tien calmura.

- El vendaval saca los peces de la mar; el nordeste nin los saca nin los mete.

- Nordeste duraor, a la tardi quedaor y a la mañana madrugaor.

- Nordeste oscuro, sur apurro.

- Nordeste que a la marea non vien, trampa tien.

- Pa Galicia nordeste y pa Asturias vendovales.

- Si el tiempu entra del noroeste y al nordeste se te rola, non esperes más que mal tiempu, con el agua hasta la bola.

4.4.6. Montañas con capa, toca, sombrero

Bajo la asignación de Montañas con capa, toca o similares, nuestra base de datos atesora 267 fichas de refranes; 20 del asturianoleonés. Tal abundancia de paremias relativas a montañas cubiertas o tocadas de nubes, con sombrero o con humanizaciones semejantes, revela una esencial mirada común sobre dicho fenómeno atmosférico, que se singulariza en los topónimos correspondientes. Paradójicamente, en cada lugar se siente el refrán propio como algo familiar y exclusivo, por más que todos ellos responden a patrones de amplio alcance románico (Pedrosa, 2001). Transcribo aquí los que contienen capa, to(u)ca y montera.

\subsubsection{Capa}

Cuando 'l picu de Moutas toma la capa, pesca la tuya y escapa; Cuando 'l 
picu Pelitrón pon capa, non dexes la tuya 'n casa; Cuando 'l picu Pierzu pon capa, coge la tuya y escapa; Cuando 'l Sueve ponga capa, non dexes la tuya ' casa; Cuando Barguero pon capa, non dexes la tuya 'n casa; Cuando Cotovellosu pon la capa, pon la tuya y escapa; Cuando Courio pon la capa, pon la tuya y escapa; Cuando la cuesta del Naranco tien capa, non dexes la tuya 'n casa; Cuando Monte Lloy pon capa, non dexes la tuya 'n casa; Si la Madalena tien capa, non dejes la tuya'n casa; si la Madalena no la tien, deja la tuya también.

\subsubsection{To(u)ca}

El DGLA (s. v. toca, la) localiza en Quirós la acepción 6: «Niebla que aparece detrás de un picacho o peña». Detrás o encima, el caso es que las montañas de referencia llevan puesta su toca o touca (según la adscripción dialectal) en estos dos refranes: Cuando Sant'Ana pon la touca, agua y no pouca; recogido por García (pp. 197, 551) en Las Dueñas (Cuideiru); Si peña Ubiña pon la toca, ;ay de nosotras!

\subsubsection{Montera}

- Cuando pon la montera el cantu Manil, Vuélvete venir [sic].

- Va llover, que peña Biguera ya pon la montera.

4.4.7. Neblina o nubes para el mar / para el monte

Es característica de tierra próxima al mar una serie de refranes en que monte y mar, como referencias para la dirección de neblinas o nubes, transmiten una predicción contrapuesta. Son refranes que materializan la previsión mediante la posibilidad, o no, de llevar a cabo las tareas agrícolas tradicionales de la labranza o la siembra:

- Cuando la neblina va del monte pa la mar, coge'l arao y vete a tchabrar, y cuando va de la mar pal monte, mete'l arao a reporte.

- Cuando la neblina vien de la mar pal monte, mete los gües ena corte; y si la neblina vien de la tierra pa la mar, saca los gües y vete arar.

- Cuando la ñieblina va del monte pal mar, unce los güés y ponte a sembrar, y cuando la ñieblina va del mar pal monte[,] desunce los gües [sic] y mételos ena corte (Castañón, 1963, p. 256).

En algunos la referencia de tierra adentro no es el monte, sino el puerto (de montaña), pero la estructura y la motivación se mantienen:

- Cuando les nubes van pa la mar, coge los gües y ponte arar, y cuando van pal Puerto[,] coge los gües y dales pienso.

- Cuando les nubes van pa la mar, cueye los gües y ponte arar; cuando las 
nubes van pal puerto, cueye 'l arau y métete dientro.

- Cuando les nubes van pa la mar, unce les vaques y ponte arar; cuando van pal Puertu, desunce les vaques y ponte a techu.

Castilla es la referencia opuesta al mar en este otro: Cuando les nubes van pa la mar, cueye los gües y ponte arar, y cuando van pa Castilla coge los gües y ponte a capilla.

Y se aleja del mar, pero no de la estructura básica, el que Castañón (1962a, p. 78) localiza en el Valle Gordo (León): Cuando los nublaus van para Asturias, aqui quedan las lluvias; cuando los nublaus van para el Bierzo, aquí queda el buen tiempo.

Por último, se hallan ecos de este tipo de refrán en portugués: As nuvens da serra para o mar, prende os bois e vai trabalhar; Nuvens do mar para a serra, põe-te ao lume com uma boa panela. Asimismo en italiano: Quando le nuvole vanno al mare [/] prendi la zappa e vai a zappare; [/] quando le nuvole vanno al monte [/] prendi gli arnesi e va' alla corte; Quando le nuvole vanno al mare, prendi la zappa e va a zappare; quando le nuvole vanno ai monti, prendi il libro e va a fare $i$ conti. Y se documentan variantes de estos en otras variedades italorromances.

\section{CONCLUSIONES}

La identificación de paremiotipos básicos sugiere la viabilidad de su plasmación en el espacio romance, por más que las correspondientes áreas parémicas no sean fácilmente delimitables, al menos con la nitidez con que cabe precisar la extensión de fenómenos relativos a elementos fonético-fonológicos, morfológicos o léxico-semánticos. Es lógico, pues las paremias, hechas de palabras, son entidades mucho más complejas que las palabras. Bajo la abstracción del paremiotipo, necesariamente flexible, los refranes en él implicados pueden reflejarse en el espacio solo de manera orientativa, a manera de manchas de aceite de contornos difusos o indefinidos. Difícilmente hallaremos, pues, isoglosas parémicas nítidas.

Y aun a sabiendas de tales dificultades de base, el equipo de ParemioRom decidió sacar partido de la geolocalización de refranes para mostrar el reflejo areal de algunos paremiotipos romances selectos. De ahí surgió el mencionado (cfr. 1.) Atlas de ParemioRom (APR), que reúne 67 paremiotipos, algunos de los cuales contienen subparemiotipos, hasta sumar un total de 121 entre unos y otros, junto a los correspondientes mapas de síntesis.

Tomaremos como ejemplo el número 4 del atlas, que responde a la fórmula «[Si / cuando] la Candelaria llora [llueve] $\rightarrow$ invierno fuera; [si / 
cuando] la Candelaria ríe [sol] $\rightarrow$ invierno por venir», en que se amplía la del paremiotipo tratado bajo el epígrafe de 4.1. Pues bien, tras el análisis y la transcripción de refranes (quedan al margen, por desgracia, los no cartografiados del aragonés), el mapa de síntesis (figura 2) refleja las correspondientes localizaciones, puntuales o zonales, y la representación asturianoleonesa en su contexto peninsular (extensible aquí al catalán insular).

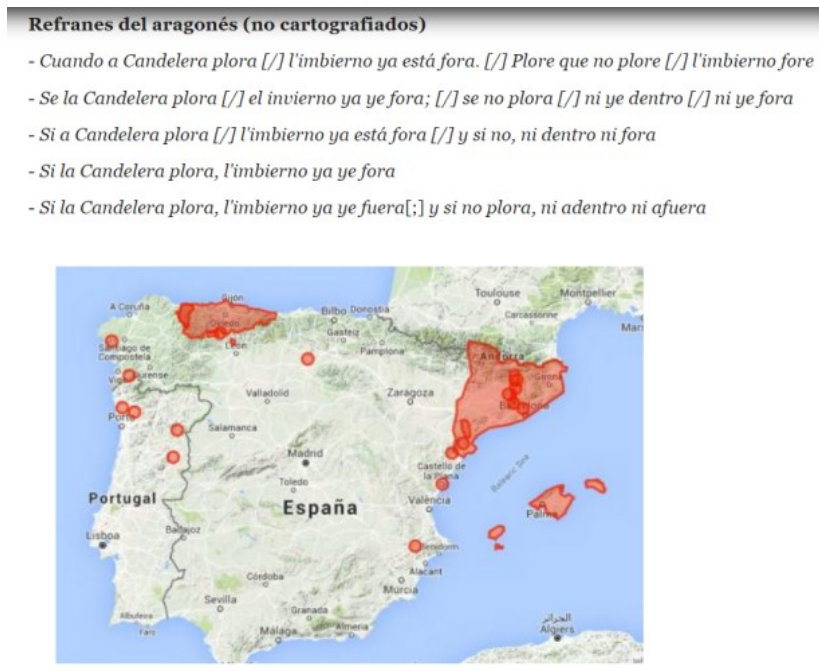

Figura 2

La cosecha del APR se podría ampliar de manera indefinida, a la carta, pues las búsquedas combinadas en la base de datos (textual, por lenguas, temática, por fuentes y por geolocalizaciones) generan automáticamente el reflejo visual de mapas de síntesis.

De ese modo, sería factible visualizar, por ejemplo, los casos de continuidad parémica del asturianoleonés con el oeste peninsular, con el gallego y con el portugués (En Xeneiro súbete al outeiro: vid. 4.2.1.); la participación en otros tipos iberorromances ( mayu / sayu: vid. 4.2.4. y 4.3.2.); o el encaje en un continuo romance general (Si llueve 'n Agostu: vid. 4.2.6.; Septiembre $\rightarrow$ puentes / fuentes: 4.2.7.). En relación con este último caso, he anticipado bajo el epígrafe 4.2.7. el rótulo de Romania parémica continua, con el que pretendo ampliar y moldear el clásico de Romania continua (Amado Alonso, 1974³ $\mathrm{p}$. 98). Así como este filólogo navarro excluía el francés y el rumano de su Romania continua, nuestro continuum parémico romance incluye el francés y excluye el rumano. A pesar del carácter apartadizo de la lengua francesa en cuanto a su fisonomía general, en lo parémico y etnográfico va con la Romania territorialmente continua, y con las islas anexas. No así el rumano, de romanicidad muy distinta en todos los órdenes, incluido el parémico y el folclórico. 
Consideración aparte merece lo específico del ámbito asturianoleonés, que también se hace visible en ParemioRom, si bien de manera testimonial: así, en el paremiotipo de 4.2.2. (Febrero $\rightarrow$ miraero), para el que no se ha hallado eco en otras lenguas o variedades románicas.

En suma, la romanicidad del asturianoleonés en su contexto romance, con mayor representación de Asturias que del resto del dominio lingüístico, y también algún caso de especificidad, se ofrecen a la sociedad y a la comunidad científica a través de la base de datos de ParemioRom desde el enfoque de la paremiología románica $\mathrm{y}$, más concretamente, desde la vertiente que en nuestro equipo investigador (Bastardas et alii 2012) dimos en llamar geoparemiología romance.

\section{Bibliografía}

ALEANR = Alvar, M., con la colaboración de A. Llorente, T. Buesa y E. Alvar (1979-1980). Atlas lingüístico y etnográfico de Aragón, Navarra y Rioja. (12 vols.). Zaragoza \& Madrid: Diputación Provincial de Zaragoza \& CSIC.

ALECant = Alvar, M., con la colaboración de J.A. Mayoral, C. Alvar, M. ${ }^{a}$ P. Nuño, M. ${ }^{a}$ C. Caballero, J. B. Corral y E. Alvar (1995). Atlas lingüistico y etnográfico de Cantabria. (2 vols.). Madrid: Arco Libros.

ALeCMan = García Mouton, P. y Moreno Fernández, F. (dirs.) (2003-). Atlas Lingüístico (y etnográfico) de Castilla - La Mancha. Universidad de Alcalá. < http://www.linguas.net/alecman/>.

$\mathrm{ALGa}=$ García, C. y Santamarina, A. (dirs.) (2003). Atlas Lingüístico Galego. Volume IV. Léxico. Tempo atmosférico e cronolóxico. A Coruña: Fundación Pedro Barrié de la Maza.

Alonso, A. (1974³). Partición de las lenguas románicas de Occidente. En Estudios lingüísticos. Temas españoles (pp. 84-105). Madrid: Gredos.

Amades, J. (1951). Folklore de Catalunya. Vol. II. Cançoner. Cançons - refranys - endevinalles. Barcelona: Editorial Selecta.

Bastardas, M-R. (2016). Lluna ajaguda, marinaru a l'addritta / luna en pie, marinâr sentât. À la recherche d'un parémiotype roman entre la lune et la mer. En N. Vuletić, X. A. Álvarez Pérez y J. E. Gargallo Gil (eds.), Mari romanzi, mari del contatto: lessico e paremiología (pp. 245256). Zadar: Sveučilište u Zadru.

Bastardas, M-R., Carpitelli, E., Fontana i Tous, J., Gargallo Gil, J. E. y Torres Torres, A. (2012). Géolinguistique 13. [Número monográfico sobre geolingüística romance].

Bastardas, M-R. y Gargallo Gil, J. E. (2016). Refranes meteorológicos con «cielo aborregado»e imágenes afines en gallego y en romance. En E. Corral Díaz, E. Fidalgo Francisco y P. Lorenzo Gradín (eds.), Cantares de amigos. Estudos en homenaxe a Mercedes Brea (pp. $97-$ 108). Santiago de Compostela: Universidade de Santiago de Compostela.

Bibliotheca Sanctorum (1961-1970). (12 vols.). Roma: Istituto Giovanni XXIII della Pontificia Università Lateranense.

Cano González, A. Ma (1989). Notas de Folklor Somedán. Uviéu: Academia de la Llingua Asturiana.

Castañón, L. (1962a). Refranero asturiano. Oviedo: Diputación de Oviedo \& Instituto de Estudios Asturianos (CSIC).

Castañón, L. (1962b): Los meses en el refranero asturiano. Revista de Dialectología y Tradiciones Populares, 18, 395-415.

Castañón, L. (1963). Refranero asturiano. Apéndice. Boletín del Instituto de Estudios Asturianos, $49,251-280$. 
Correas Martínez, M. y Gargallo Gil, J. E. (2002). Ya entra el sol por las umbrías. Altura y proyección del sol en el calendario romance de refranes. Paremia, 11, 21-30.

Correas Martínez, M. y Gargallo Gil, J. E. (2003). Calendario romance de refranes. Barcelona: Publicacions i Edicions de la Universitat de Barcelona.

DALLA = Academia de la Llingua Asturiana (2015). Diccionariu de la Llingua Asturiana. Versión electrónica: $<$ http://www.academiadelallingua.com/diccionariu/ $>$.

DCECH = Coromines, J., con la colaboración de J. A. Pascual (1980-1991). Diccionario crítico etimológico castellano e hispánico. (6 vols.). Madrid: Gredos.

DCVB = Alcover, Antoni M. / Moll, Francesc de B. (1930-1962). Diccionari català-valenciàbalear. (10 vols.). Palma de Mallorca: Editorial Moll. [También accesible en línea: http://dcvb.iecat.net/].

DGLA = García Arias, X. Ll. (2002-2004). Diccionario General de la Lengua Asturiana . Oviedo: Editorial Prensa Asturiana S.A. / La Nueva España. [Accesible en línea: $<$ https://mas.lne.es/diccionario/>].

DIEC = Institut d'Estudis Catalans $\left(2007^{2}\right)$. Diccionari de la llengua catalana. [Versión en línea: $<$ https://dlc.iec.cat/>].

DLE = Real Academia Española (2014). Diccionario de la lengua española. Barcelona: Espasa. [También accesible en línea: http://dle.rae.es/].

Fontana i Tous, J. y Gargallo Gil, J. E. (2018). Refranes meteorológicos y del calendario en la encuesta digital Els refranys més usuals de la llengua catalana. Paremia, 27, 37-47.

García García, L. (1976). Santos y fiestas en el refranero astur. Boletín del Instituto de Estudios Asturianos, 88-89, 535-563.

Gargallo Gil, J. E. (2003). Més ençà o mès enllá, la Quaresma en març caurà. Refranes romances del mes de marzo. Paremia, 12, 41-54.

Gargallo Gil, J. E. (2004). Dos de febrero. Refranes romances de la Candelaria y meteorología popular. Paremia, 13, 109-124.

Gargallo Gil, J. E. (2006). Reseña del DGLA. Estudis Romànics, 28, 381-386.

Gargallo Gil, J. E. (2007a). Garda o teu saio para maio. Consellos de abrigo no calendario romance de refráns. Cadernos de Fraseoloxía Galega, 9, 95-112.

Gargallo Gil, J. E. (2007b). La lluna setembrina, set llunes endevina. Refráns meteorolóxicos con lúa na Romania. En G. Conde Tarrío (dir.), El componente etnolingüistico de la Paremiología. The ethnolinguistic Component of Paremiology. (pp. 117-134). Fernelmont: Editions Modulaires Européennes (E.M.E.).

Gargallo Gil, J. E. (2010). Refranes del calendario y meteorológicos en el Diccionario General de la Lengua Asturiana (DGLA). En A. Ma. Cano González (ed.), Homenaxe al Profesor Xosé Lluis García Arias (tomo I, pp. 177-193). Uviéu: Academia de la Llingua Asturiana.

Gargallo Gil, J. E. (2018a). Fins a coranta d'es bril... Quaranta i quarantenes al calendari de refranys. Estudis Romànics, 40, 259-266.

Gargallo Gil, J. E. (2018b). Hasta el cuarenta de mayo... Referencias al 'cuarenta' en los refranes romances del calendario. eHumanista/IVITRA, 14, 862-881.

Gargallo Gil, J. E. y Fontana i Tous, J. (2018). Chámanlle á lúa Lucía, chámanlle ó sol Manoel... Refranes meteorológicos, romances con sol y luna. En X. A. Álvarez Pérez y Mercedes Brea (eds.), Obreiro da lingua, amigo da xente. Estudos de xeografia lingüística en homenaxe a Manuel González (pp. 153-163). Santiago de Compostela: Universidade de Santiago de Compostela.

Gargallo Gil, J. E. y Ruiz-Zorrilla Cruzate, M. (2012). El concepto de veranillo en Europa. Ensayo semántico-motivacional. Géolinguistique, 13, 135-158.

Gomis, C. (1998). Meteorologia i agricultura populars. Recull d'aforismes, modismes, creences i supersticions referents a la meteorologia i a l'agricultura a l'entorn dels anys 1864 a 1915. Edició notablement augmentada amb gran nombre de confrontacions a cura de Cels Gomis $i$ Serdañons. Barcelona: Alta Fulla.

Manent, A. (1997). Els noms populars de núvols, boires $i$ vents: Ribera d'Ebre $i$ Terra Alta. 
Barcelona: Centre d'Estudis Riudomencs «Arnau de Palomar».

Martín-Vide, J. (2011). ¿Qué tienen de verdad los refranes meteorológicos? En J. E. Gargallo Gil, M-R. Bastardas, J. Fontana i Tous, G. Iannàccaro y Antonio Torres Torres (eds.) (pp. 247258), I proverbi meteorologici. Ai confini dell'Europa romanza. Alessandria: Edizioni dell'Orso.

Martínez Kleiser, L. (1945). El tiempo y los espacios de tiempo en los refranes. Madrid: Librería General de Victoriano Suárez.

Mieder, W. (1996). Los refranes meteorológicos. Paremia, 5, 59-65.

Palacios Rasal, L. y Baos Muñoz, Ch. (2010). Bocabulario de Murillo de Galligo. Uesca: Publicazions d'o Consello d'a Fabla Aragonesa.

Pedrosa Bartolomé, J. M. (2001). El gorro de Montejurra (un estudio de paremiología comparada). Cuadernos de etnología y etnografía de Navarra, 76, 149-158.

Rodríguez Marín, F. (1896). Los refranes del almanaque. Recogidos, explicados y concordados con los de varios paises [sic]. Sevilla: Imp. de Francisco de P. Díaz.

Rúa Aller, F. J. (2014). Refranes del tiempo en León. León: Eolas.

Sanchis Guarner, M. (1951). Calendari de refranys. Barcelona: Barcino.

Spano, G. (1997 [1871]). Proverbi sardi trasportati in lingua italiana e confrontati con quelli degli antichi popoli. A cura de Giulio Angioni. Nuoro: Ilisso.

Tagliavini, C. (1963). Storia di parole pagane e cristiane attraverso i tempi. Brescia: Morcelliana.

Vázquez Saco, F. (2003). Refraneiro galego e outros materiais de tradición oral. Santiago de Compostela: Xunta de Galicia \& Consellería de Educación e Ordenación Universitaria \& Centro Ramón Piñeiro para a Investigación en Humanidades. [Cadernos de Fraseoloxía Galega, 5].

Recibíu: 17.12.2019

Aceutáu: 13.4 .2020 